\title{
Planetary Nebulae Surveys Beyond the Local Group
}

\author{
Ortwin Gerhard ${ }^{1}$ \\ ${ }^{1}$ Max-Planck-Institut für Extraterrestrische Physik, \\ Giessenbachstrasse, D-85741 Garching, Germany \\ email: gerhard@mpe.mpg.de
}

\begin{abstract}
Distant planetary nebulae (PNe) are used to measure distances through the PN luminosity function, as kinematic tracers in determining the mass distribution in elliptical galaxies, and most recently, for measuring the kinematics of the diffuse stellar population in galaxy clusters. This article reviews the photometric and spectroscopic survey techniques that have been used to detect PNe beyond the Local Group, out to the Coma cluster at $100 \mathrm{Mpc}$ distance. Contaminations by other emission sources and ways to overcome them will be discussed as well as some science highlights and future perspectives.
\end{abstract}

Keywords. planetary nebulae, surveys, photometry, spectroscopy, distances, elliptical galaxies, dark matter, galaxy clusters, intracluster light

\section{Introduction}

The search for planetary nebulae (PNe) beyond the Local Group is motivated by several important science projects:

- The maximum luminosity of a PN in the [O III] $\lambda 5007$ line varies by at most $0.1-0.2$ mag between the stellar populations of a variety of galaxies. The PN luminosity function (PNLF), in particular its bright cutoff, is therefore an important distance estimator (see Jacoby 1989, Ciardullo et al. 1989, Jacoby, Ciardullo, Ford 1990, and the review by Ciardullo 2003).

- The radial velocities of PNe, also measured from the redshifted [O III] line, can be used to trace the kinematics of stellar populations that have too low surface brightness for other kinematic measurements. PNe are therefore used as tracers of the mass distribution in elliptical galaxies at intermediate radii $\left(2-5 R_{e}\right)$, where measuring radial velocities from integrated absorption line spectra is no longer feasible (Hui et al. 1995, Arnaboldi et al. 1996, 1998, Méndez et al. 2001, Romanowsky et al. 2003, Peng, Ford \& Freeman 2004).

- PNe are currently the only tracers (perhaps excepting globular clusters) that allow us to measure the kinematics of the diffuse stellar population in galaxy clusters, and thus to investigate the dynamics and cosmological origin of this population (Arnaboldi et al. 2004, Gerhard et al. 2005).

$\mathrm{PNe}$ have been detected out to distances of some $20 \mathrm{Mpc}$ with narrow-band or slitless spectroscopy surveys, and out to $100 \mathrm{Mpc}$ (the Coma cluster) with multislit imaging spectroscopy (MSIS) surveys. Sections 2-4 contain short discussions of these survey techniques. The final Section 5 describes a few recent scientific highlight results based on PN surveys and gives a brief outlook on future work. 


\section{Photometric surveys}

Optical spectra of Galactic PNe show numerous emission lines but essentially no continuum flux. The strongest emission line is [O III] $\lambda 5007$, which contains $\sim 10 \%$ of the energy output of the central star. Further strong lines are the second [O III] $\lambda 4959$ line (three times weaker than $\lambda 5007)$, and $\mathrm{H} \alpha$ (3-5 times weaker). In spectra of $\mathrm{PNe}$ at the Virgo distance, it needs an $8 \mathrm{~m}$ telescope to clearly see both lines of the [O III] doublet; such spectra only became available recently (Arnaboldi et al. 2003, 2004).

Blinking surveys Based on the lack of continuum and the strong [O III] $\lambda 5007$ emission line, Ciardullo, Ford, Jacoby and collaborators (e.g., Ciardullo et al. 1989, Jacoby, Ciardullo, Ford 1990) developed the so-called on-band/off-band survey technique for distant PNe. This involves imaging the field of interest with a narrow-band filter around the $[\mathrm{O}$ III $]$ line and with a broad-band filter away from the dominant emission lines. In the early surveys the PN candidates were identified by blinking the two images. The PN candidates are those point sources in the narrow-band image that have no counterpart in the broad-band image. Once identified, the [O III] fluxes of the PN candidates were determined by aperture photometry, and converted to $m_{5007}=-2.5 \log F_{5007}-13.74$ magnitudes.

As the early surveys were mostly targeted at elliptical galaxies (in order to derive PNLF distances), contamination of the PN samples by $\mathrm{H}$ II regions and background galaxies was largely absent: the surface density of PNe in elliptical galaxies is much greater than the surface density of background galaxies whose redshifted Ly $\alpha$ or [O II] emission lines fall into the [O III] filter. To avoid contamination by Galactic stars, the off-band image was taken to be typically $\sim 0.25 \mathrm{mag}$ deeper than the on-band image (see also $§ 2.2$ ). Nonetheless, at least one such sample contained a substantial fraction of candidates that were not confirmed by later work (see Arnaboldi et al. 2003).

The number density distributions of PN candidates in elliptical galaxies were found to approximately follow the galaxy light, indicating constant $\alpha_{B}$, the ratio of PNe to B-band luminosity. Inside some radius, of order the effective radius $R_{e}$, however, the number density profile flattens off, as fainter PNe are increasingly lost against the bright galaxy background. This effect is present also in more recent slitless surveys in ellipticals. Construction of the PNLF therefore requires identification of a homogeneous and complete PN sample, which excludes the central regions, as well as PNe fainter than a magnitude limit set such that the remaining $\mathrm{PNe}$ are detected with signal-to-noise of at least 9 per pixel (in simulations, essentially all objects are detected at this $\mathrm{S} / \mathrm{N}$ level).

Wide-field surveys Following the discovery of three intergalactic PNe in the Virgo cluster near M86 (Arnaboldi et al. 1996), wide-field imaging (WFI) surveys were undertaken to map this much more sparse PN population in Fornax, Virgo, and several galaxy groups (Feldmeier, Ciardullo \& Jacoby 1998, Arnaboldi et al. 2002, 2003, Ciardullo et al. 2002a, Feldmeier et al. 2003, Castro-Rodríguez et al. 2003, Aguerri et al. 2005). Again, images are taken in a narrow-band filter around the red-shifted [O III] $\lambda 5007$ line, typically $\simeq 60 \AA$ wide to cover the wider cluster velocity distribution, and in a nearby broad-band filter. Such WFI fields contain many 1000's of stars, so the blinking method is no longer feasible and automatic methods for sifting out the PNe must be used. One such technique is based on the narrow-band vs. broad-band colour magnitude diagram (CMD), as pioneered by Theuns \& Warren (1997). An example for the Virgo RCN1 field from Arnaboldi et al. (2002) is shown in Figure 1. PNe candidates in this field are those to the right of the diagonal line indicating the continuum limit, and below the other lines to exclude Galactic stars and background emission galaxies with EW below $110 \AA$, as described in the figure caption. Because of the much lower surface density of PNe 


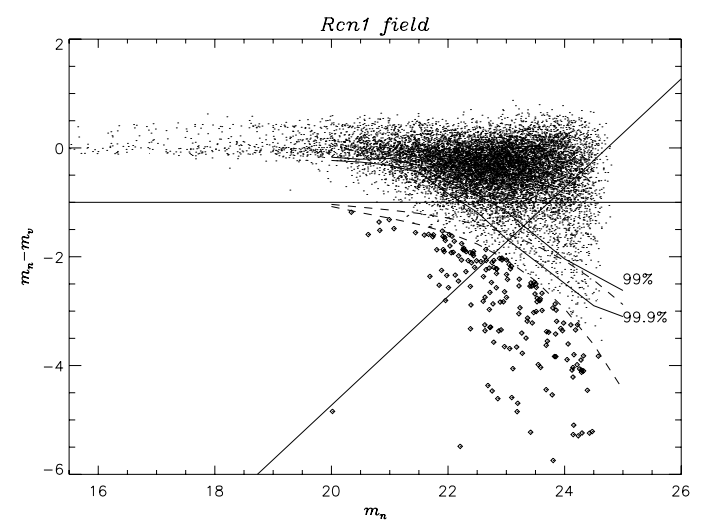

Figure 1. Color-magnitude diagram for all the unresolved sources in the Virgo RCN1 field. The horizontal line at $m_{n}-m_{v}=-1$ indicates objects with an observed $\mathrm{EW}=110 \AA$. The diagonal line shows the magnitude corresponding to $1.0 \times \sigma$ above sky in the $\mathrm{V}$ band. Full curved lines represent the $99 \%$ and $99.9 \%$ lines for the distribution of modelled continuum objects. The dashed lines represent $84 \%$ and $97.5 \%$ lines for the distribution of modelled objects with $m_{n}-m_{v}=-1$. The points are all objects detected by SExtractor. Diamonds are objects with a significant color excess in the narrow band filter. From Arnaboldi et al. (2002).

in these intracluster fields, the PN catalogues extracted from the images may contain a substantial fraction of contaminants. We now briefly discuss these various interlopers and how to avoid them.

Ordinary Galactic stars. In Fig. 1, stars were excluded from the PN catalogue by considering their $m_{n}-m_{b}$ colour distribution after convolution with the photometric errors and discarding all objects above the $99.9 \%$ line. However, the photometric errors may conspire to result in a bright narrow-band $m_{n}$ and faint broad-band $m_{b}$, so as to result in apparently large EW (negative colour) and undetectable continuum, even for stars with $\mathrm{AB}$ colour zero (requiring sufficiently large negative error in $m_{n}$ and positive error in $m_{b}$ ). Such errors move stars from the top right of Fig. 1 into the region of the diagram containing the high-EW sources. While statistically not very likely, for $\sim 10^{4}$ stars in the image this "spill-over" effect (Aguerri et al. 2005) may occur often enough to lead to a significant contamination of the PN samples. From the simulations for their fields, Aguerri et al. (2005) found that $60 \% / 20 \% / 10 \%$ of their Virgo intracluster PN (ICPN) samples were stars due to the spillover effect, if the limiting magnitude of the off-band image was 0/0.5/0.9 mag deeper than the limiting magnitude of the on-band image. Thus the contamination by faint stars may be avoided by using sufficiently deep off-band images.

$\mathbf{H}$ II regions. Imaging surveys including $\mathrm{H} \alpha$ have found a few compact $\mathrm{H}$ II regions in one Virgo field (Gerhard et al. 2002, Arnaboldi et al. 2003), probably associated with gas that was stripped recently from galaxies, but this is a small fraction of all sources in the ICPN catalogue.

$[\mathrm{O}$ II] emission galaxies at redshift $\simeq 0.35$, whose $[\mathrm{O}$ II $]$ emission lines fall into the [O III] narrow-band filter at Virgo redshift. The contamination from these objects can be greatly reduced by retaining only emission sources with observed EW greater than $100 \AA$ : Hammer et al. (1997) and Hogg et al. (1998) found no [O II] emitters with restframe EW $>70 \AA$. This has been confirmed by spectroscopic follow-up observations of Virgo ICPNe candidates, none of which showed the [O II] doublet and a continuum (Arnaboldi et al. 
2004). Of course, such a stringent EW cut also eliminates some PNe from the catalogue, for which the emission is too weak compared to the sky noise.

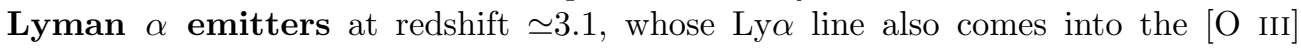
filter. This class of contaminants was found originally by Kudritzki et al. (2000) taking spectra of ICPN candidates in a Virgo field which indeed is consistent with containing no intracluster light (ICL; Aguerri et al. 2005, Mihos et al. 2005). Subsequent studies of Ly $\alpha$ emitters in blank fields have shown that the Ly $\alpha$ luminosity function is spatially variable and that the brightest Ly $\alpha$ emitters are between $0.5-1.5$ magnitudes fainter than the PNLF cutoff in Virgo (Ciardullo et al. 2002b, Castro-Rodríguez et al. 2003, Aguerri et al. 2005). With good spatial resolution extended Ly $\alpha$ galaxies may be eliminated, but according to the simulations of Aguerri et al. (2005) typically $\sim 25 \%$ of the ICPN candidates in Virgo could be Ly $\alpha$ background galaxies.

A large fraction of these will be recognized in follow-up spectroscopy by their continuum, their broad emission lines, or by the absence of [O III] $\lambda 4959$ if this is in the spectral range. The spectroscopic results of Arnaboldi et al. (2004) have confirmed $80 \%$ of the expected number of PNe in the recent Virgo photometric surveys as real PNe, showing that these surveys are now well-understood. Many of the confirmed Virgo ICPNe also show the second [O III] $\lambda 4959$ line besides [O III] $\lambda 5007$, confirming without doubt that these objects are in the cluster.

The measured radial velocities then provide us with the kinematics of the intracluster stellar population; see the scientific highlight in Section 5. Similarly, the combination of photometric survey plus spectroscopic follow-up has been used to obtain samples of PN radial velocities in the halos of elliptical galaxies. See the references in the Introduction.

\section{Slitless spectroscopy surveys}

Dispersed/undispersed imaging In this technique, pioneered by Méndez et al. (2001), undispersed images of the field are taken both through a broad band filter and through a narrow band filter centered around the redshifted [O III] $\lambda 5007$ line for the target PNe. The PN candidates are identified as those sources that appear only in the onband image, which also provides accurate astrometric positions. Additionally, a dispersed image is taken through a grism and the on-band filter. In this image, the PN candidates are still point sources, but are now shifted relative to their true positions on the sky by an amount reflecting both the mapping by the grism and their radial velocities. By contrast, stars in the field now appear as elongated features on the CCD, with length corresponding to the filter width.

From such data in two overlapping fields, Méndez et al. (2001) obtained fluxes and radial velocities for $535 \mathrm{PN}$ candidates in the E5 galaxy NGC 4697, with velocity errors of $\sim 40 \mathrm{~km} \mathrm{~s}^{-1}$ and magnitude errors of $0.1-0.2 \mathrm{mag}$ for the bright/faint sources. Because this is a spectroscopic technique, there are fewer contaminants than in the imaging surveys; only background galaxies with faint continuum and narrow emission line will remain unrecognized. Méndez et al. obtained a new PNLF distance to NGC 4697 of $D \simeq 10.5 \mathrm{Mpc}$, and found that the falling velocity dispersion profile in this galaxy is consistent with simple isotropic spherical models without dark matter halo out to $\sim 2.5 R_{e}$.

A similar analysis was done by Teodorescu et al. (2005) for the Fornax elliptical galaxy NGC 1344. However, in this galaxy the velocity dispersion profile is not consistent with isotropic constant-M/L models, so most likely some dark matter is indicated. For more details on this method and the results obtained, see Méndez, this conference.

Counter-dispersed imaging (CDI) The CDI technique instead uses two images dispersed in opposite directions, to determine both the true positions of the PN candidates 
on the sky and their radial velocities. This technique is employed by the special-purpose PN.S instrument mounted at the WHT at La Palma, in which the incoming beam, after passing the [O III] $\lambda 5007$ filter, is split into two parts that are simultaneously imaged through separate grisms in two [O III] arms. (A third $\mathrm{H} \alpha$ arm is currently being built that will also allow simultaneous $\mathrm{H} \alpha$ imaging of the field). Two noteworthy properties of the PN.S instrument are its wide $\left(11 .^{\prime} 4 \times 10 .^{\prime} 3\right)$ field of view and high system efficiency (33\%); see Douglas et al. (2002). With the $\mathrm{H} \alpha$ arm, it will also be possible to remove highredshift galaxies with narrow emission line and faint continuum, which are the remaining contaminants in the current samples, similar to the Méndez et al. (2001) technique.

First results based on PN.S data for three intermediate-luminosity elliptical galaxies were published by Romanowsky et al. (2003). Like Méndez et al. (2001) for NGC 4697, they found that all three galaxies (NGC 821, 3379, 4494) have falling dispersion profiles out to several $R_{e}$, which are consistent with dynamical models with little if any dark matter out to these radii. The PN.S has also been highly successful in producing a sample of some 2500 PNe in M31 (Merrett et al. 2006). Currently, most of the data for the PN.S team's core science program have been taken, and are being analyzed with an improved data pipeline and revised dynamical models. The goal of the core science program is to study a sample of 12 round elliptical galaxies and obtain constraints on their dark matter halos out to $\sim 6 R_{e}$ (for more details, see Romanowsky, this conference).

\section{Multi-slit imaging spectroscopy surveys}

Beyond about 20-30 Mpc distance, PNe are too faint to be detectable with narrow band surveys or slitless spectroscopy - their emission disappears in the sky noise in the narrow band filter. The brightest PNe in the Coma cluster at $100 \mathrm{Mpc}$ distance have line fluxes of $2.2 \times 10^{-18} \mathrm{erg} \mathrm{s}^{-1} \mathrm{~cm}^{-2}$ - this is equivalent to $\sim 20$ photons per minute through the aperture of an $8 \mathrm{~m}$ telescope, of which $\sim 2$ will reach the detector for a typical $\sim 10 \%$ overall system efficiency. To detect PNe at such distances requires a spectroscopic blind search technique: spectroscopic, so that only the sky noise within a few $\AA$ dilutes the emission from the PN, and blind, because the positions of these faint PNe cannot be previously determined.

The Multi-Slit Imaging Spectroscopy (MSIS) technique (Gerhard et al. 2005) which meets these requirements combines a mask of parallel multiple slits with a narrow-band filter, centered on the redshifted [O III] $\lambda 5007$ emission line. Spectra are obtained of all PNe that lie behind the slits. The narrow band filter limits the length of the spectra on the CCD so that many slits can be simultaneously exposed. For each set of mask exposures only a fraction of the field is surveyed; to increase the sky coverage the mask can be stepped on the sky. The approach is similar to some searches for high $z>5 \operatorname{Ly} \alpha$ emitters (e.g., Tran et al. 2004).

In their pilot study, Gerhard et al. (2005) obtained MSIS observations of the Bernstein et al. (1995) field in the Coma cluster core. This field is at the center of the cluster X-ray emission and $\sim 5$ arcmin away from the $\mathrm{cD}$ galaxy NGC 4874 . From the Bernstein et al. (1995) diffuse light measurement we expected about $425 \mathrm{PNe}$ in this field. The fraction of the field surveyed by the mask was $\sim 12 \%$; because $\sim$ half of the PNe will be dimmed as they are not centered in the slits, we estimated to detect $\sim 20-30$ PNe per mask.

In the MSIS setup, a compromise must be found between the number of slits and hence number of objects detected, and the velocity resolution and signal-to-noise (S/N) of the emission sources, which are dependent on the spectral resolution. For the Coma observations, a mask with 70 slits was used, each spectrum was 43 pixels on the CCD, and the spectral resolution $d$ corresponded to $440 \mathrm{~km} \mathrm{~s}^{-1}$. Then the total $\mathrm{S} / \mathrm{N}$ over all 

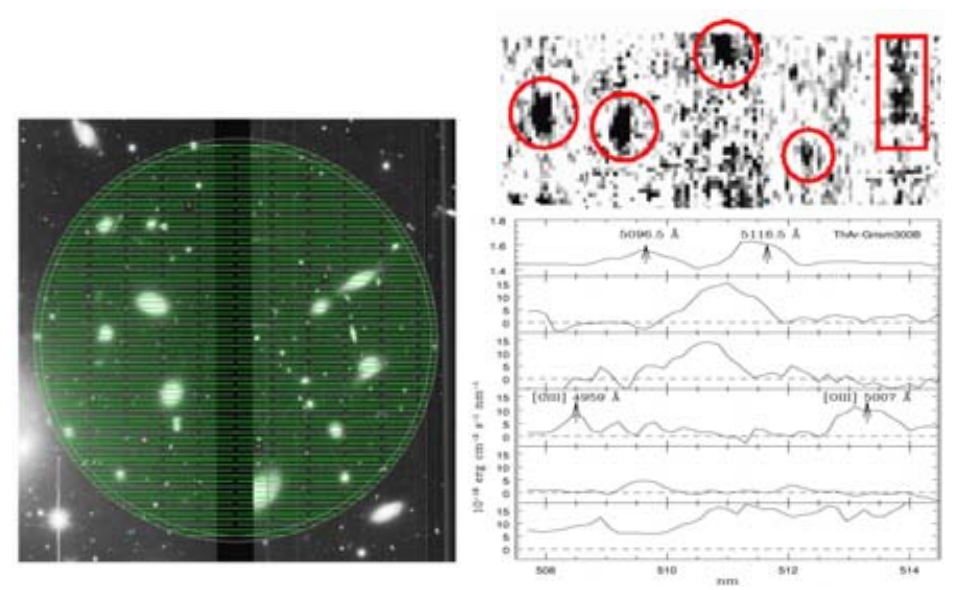

Figure 2. Left: Multi-slit mask used in the MSIS observations, superposed on the Coma core field. Right: 1D (top) and 2D (bottom) spectra of 4 PN candidates and a probable Ly $\alpha$ galaxy (right and bottom panel, respectively) in the Coma core field. Also shown is a 1D arc lamp spectrum at the spectral resolution of the setup, $7.2 \AA=440 \mathrm{~km} \mathrm{~s}^{-1}$. Note the [O III] $\lambda 4959$ line in the third PN spectrum. After Gerhard et al. (2005). [See online paper for colour version.]

pixels becomes

$$
\mathrm{SNR}_{P N}=7.0 \times 10^{-0.4 \Delta m} \times\left(\frac{d}{1.45 \AA / \mathrm{pix}}\right)^{-1 / 2}\left(\frac{\phi}{0 .^{\prime \prime} 6}\right)^{-1}\left(\frac{\theta}{6 \sigma_{y}}\right)^{-1 / 2}\left(\frac{t_{\text {exp }}}{3 \mathrm{hrs}}\right)^{1 / 2}
$$

for a PN fainter than the cutoff by $\Delta m$ magnitudes. Here $\phi$ is the seeing FWHM, $\theta$ the slit width, $\sigma_{y}$ the pixel size, and $t_{\text {exp }}$ the exposure time. Relative to the sky noise in one pixel, the corresponding $\mathrm{S} / \mathrm{N}$ of a PN at the PNLF cutoff $(\Delta m=0)$ was 32 . Both values assume that the emission from the PN falls through the slit completely. Detection of a source is normally considered secure if the $\mathrm{S} / \mathrm{N}$ relative to the sky noise in one pixel is $\geqslant 9$. In the Coma data this corresponds to $\mathrm{SNR}_{P N} \geqslant 2$, i.e., $95 \%$ probability of the source being real. If this criterion is used for secure detection, PNe about 1.4 magnitudes down the PNLF could be detected. It is clear from equation (4.1) that the most important condition for successful detection is good seeing.

Figure 2 shows the mask used in the Coma observations, as well as the two-dimensional and one-dimensional spectra of some of the PN candidates and a probable Ly $\alpha$ emitting galaxy, and of the arc lamp. The faintest candidate in Fig. 2 has a total $\mathrm{SNR}_{P N}=2.0$, corresponding to a flux of $3 \times 10^{-19} \mathrm{erg} \mathrm{s}^{-1} \mathrm{~cm}^{-2}(\simeq 68$ photons in three hours exposures with FOCAS). The reasons to believe that these emission sources are indeed almost all PNe in the Coma cluster are: (1) They are unresolved spatially and in wavelength; (2) they have no detectable continuum, down to $1.6 \times 10^{-20} \mathrm{erg} \mathrm{s}^{-1} \mathrm{~cm}^{-2} \AA^{-1}$, the flux from two $\mathrm{O}$ stars at the distance of Coma; (3) for all four bright sources for which [O III] $\lambda 5007$ is sufficiently redshifted, the second [O III] $\lambda 4959$ line has been detected in the blue part of the filter; (4) the emission fluxes are consistent with those expected for PNe in Coma, at and below the bright cutoff of the PNLF; (5) the number density and (6) the radial velocity distribution are also consistent with the surface brightness of ICL and the radial velocities of Coma galaxies in this field. On the contrary, emission sources with measured continuum (likely background galaxies) have a much more uniform velocity distribution.

These first ICPN observations in Coma have already lead the way to the conclusion that the two $\mathrm{cD}$ galaxies with their associated subclusters are currently undergoing a strong 


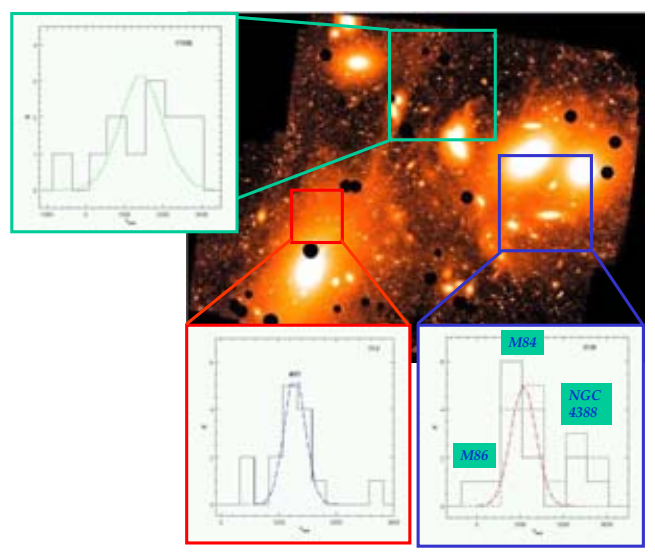

Figure 3. Montage of a deep image of the Virgo cluster core from Mihos et al. (2005), including M87 (lower left) and M86/M84 regions (right), and histograms of ICPN velocities measured by Arnaboldi et al. (2004) in the indicated fields. The image and kinematics show that M87 has an extended, dynamically bound halo. By contrast, the large halo around M84, M86, and NGC 4388 is a superposition; the PNe velocities in this field can clearly be associated with the individual galaxies, but M84 also has an extended, dynamically bound halo. The streamers in the image as well as the ICPN velocities in the Core and M87 fields show that the intracluster stars in Virgo have not yet mixed to virial equilibrium. [See online paper for colour version.]

interaction prior to merging (see Arnaboldi, these proceedings). A lot more insight into the dynamics of present-day clusters and their ICL can be expected in the next few years with the MSIS technique, velocities of ICPNe can be measured in all clusters out to $\sim 100 \mathrm{Mpc}$ distance.

\section{Some recent science highlights and future prospects}

Here I will briefly mention three areas in which recent PN survey work has led to important and sometimes surprising results, and where further work will be needed.

(1) PNe population effects. A reanalysis of the Méndez et al. (2001) sample of 535 PNe in the elliptical galaxy NGC 4697 has shown that most of the brightest of these PNe are part of a subpopulation which is azimuthally unmixed and kinematically peculiar, and thus neither traces the distribution of all stars nor can be in dynamical equilibrium in the gravitational potential (Sambhus et al. 2006). This introduces an uncertainty in the PNLF cutoff luminosity for this galaxy of $\sim 0.15 \mathrm{mag}$; the PNLF of two kinematic subsamples differ with statistical significance. Probably related is the recent result that the bright and faint ICPNe in the MSIS Coma cluster sample (see §4) have different velocity distributions (Arnaboldi et al., in preparation).

(2) Low density dark matter halos in elliptical galaxies. The work of Méndez et al. (2001) and Romanowsky et al. (2003) based on slitless spectroscopy PN surveys has provided evidence that in some intermediate luminosity ellipticals the velocity dispersion profiles are falling out to at least several $R_{e}$, suggesting that the dark matter halos in these galaxies must be fairly diffuse. However, other ellipticals appear to have denser halos (e.g., Gerhard et al. 2001); also, further study is needed to address the concerns of Dekel et al. (2005). The PN.S key project results will be very valuable to clarify these issues.

(3) Kinematics of ICL. Figure 3, based on the results of Arnaboldi et al. (2004) and Mihos et al. (2005), gives a vivid illustration how the velocities of ICPNe can shed light 
on the kinematics and dynamics of the ICL, showing clearly the dynamical youth of the Virgo cluster. Both M87 and M84 have extended stellar halos in approximate dynamical equilibrium with their parent galaxies. Superposed on the M87 halo and in the CORE field stars are seen which clearly have intracluster kinematics but whose velocity distributions do not appear to be well-mixed yet, and in the SUB field, all of the PNe observed are consistent with belonging dynamically to the large galaxies in the field. ICPN and galaxy velocities indicate that, somewhat surprisingly, also the much denser Coma cluster is in a stage of strong dynamical evolution (Gerhard et al. 2005, Arnaboldi et al., in preparation, Adami et al. 2005). Further measurements of ICPN velocities as discussed in $\S 4$ will show whether generally the ICL in nearby galaxy clusters is inhomogeneous and dynamically evolving, or whether there are clusters in which it has already found a reasonably mixed equilibrium configuration.

Acknowledgments I would like to acknowledge the Swiss National Science Foundation for supporting the research program on PNe in distant galaxies and clusters during several grant periods.

\section{References}

Adami, C., Biviano, A., Durret, F., \& Mazure, A. 2005, A\& A, 443, 17

Aguerri, J.A.L., Gerhard, O.E., Arnaboldi, M., \& Napolitano, N. et al. 2005, AJ, 129, 2585

Arnaboldi, M., Aguerri, J.A.L., Napolitano, N.R., Gerhard, O., et al. 2002, AJ, 123, 760

Arnaboldi, M., Freeman, K.C., Gerhard, O., Matthias, M., et al. 1998, ApJ, 507, 759

Arnaboldi, M., Freeman, K.C., Mendez, R.H., Capaccioli, M., et al. 1996, ApJ, 472, 145

Arnaboldi, M., Freeman, K.C., Okamura, S., Yasuda, N., et al. 2003, AJ, 125, 514

Arnaboldi, M., Gerhard, O.E., Aguerri, J.A.L., et al. 2004, ApJ, 614, L33

Bernstein, G.M., Nichol, R.C., Tyson, J.A., Ulmer, M.P., \& Wittman, D. 1995, AJ, 110, 1507

Castro-Rodríguez, N., Aguerri, J.A.L., Arnaboldi, M., Gerhard, O., et al. 2003, A\&A, 405, 803

Ciardullo, R. 2003, Lect. Notes Phys., Springer, 635, 243

Ciardullo, R., Feldmeier, J.J., Krelove, K., Jacoby, G.H., \& Gronwall, C. 2002a, ApJ, 566, 784

Ciardullo, R., Feldmeier, J.J., Jacoby, G.H., Kuzio de Naray, R., et al. 2002b, ApJ, 577, 31

Ciardullo, R., Jacoby, G.H., \& Ford, H.C. 1989, ApJ, 344, 715

Dekel, A., Stoehr, F., Mamon, G.A., Cox, T.J., Novak, G.S., et al. 2005, Nature, 437, 707

Douglas, N.G., Arnaboldi, M., Freeman, K.C., Kuijken, K., et al. 2002, PASP, 114, 1234

Feldmeier, J.J., Ciardullo, R., \& Jacoby, G.H. 1998, ApJ, 503, 109

Feldmeier, J.J., Ciardullo, R., Jacoby, G.H., \& Durrell, P.R., 2003, ApJS, 145, 65

Gerhard, O., Arnaboldi, M., Freeman, K.C., \& Okamura, S. 2002, ApJ, 580, L121

Gerhard, O., Arnaboldi, M., Freeman, K.C., et al. 2005, ApJ, 621, L93

Gerhard, O., Kronawitter, A., Saglia, R.P., \& Bender, R. 2001, AJ, 121, 1936

Hammer, F., Flores, H., Lilly, S.J., Crampton, D., Rola, C., et al. 1997, ApJ, 481, 49

Hogg, D.W., Cohen, J.G., Blandford, R., \& Pahre, M.A. 1998, ApJ, 504, 622

Hui, X., Ford, H.C., Freeman, K.C., \& Dopita, M.A. 1995, ApJ, 449, 592

Jacoby, G.H. 1989, ApJ, 339, 39

Jacoby, G.H., Ciardullo, R., \& Ford, H.C. 1990, ApJ, 356, 332

Kudritzki, R.-P., Méndez, R.H., Feldmeier, J.J., Ciardullo, R., et al. 2000, ApJ, 536, 19

Méndez, R.H., Riffeser, A., Kudritzki, R.-P., Matthias, M., et al. 2001, ApJ, 563, 135

Merrett, H.R., Merrifield, M.R., Douglas, N.G., et al. MNRAS, 369, 120

Mihos, J.C., Harding, P., Feldmeier, J., \& Morrison, H. 2005, ApJ, 631, L41

Peng, E., Ford, H.C., \& Freeman, K.C. 2004, ApJ, 602, 685

Romanowsky, A.J., Douglas, N.G., Arnaboldi, M., Kuijken, K., et al. 2003, Science, 301, 1696

Sambhus, N., Gerhard, O., \& Méndez, R.H. 2006, AJ, 131, 837

Teodorescu, A.M., Méndez, R.H., Saglia, R.P., Riffeser, A., et al. 2005, ApJ, 635, 290

Theuns, T. \& Warren, S.J. 1997, MNRAS, 284, L11

Tran K.-V., Lilly, S.J., Crampton, D., \& Brodwin, M. 2004, ApJ, 612, L89 